\title{
Torsional Periodic Lattice Distortion in Twisted Bilayer Graphene
}

Suk Hyun Sung ${ }^{1}$, Yin Min Goh ${ }^{1}$, Hyobin Yoo $^{2}$, Rebecca Engelke ${ }^{3}$, Philip Kim ${ }^{2}$ and Robert Hovden ${ }^{1}$

${ }^{1}$ University of Michigan, Ann Arbor, Michigan, United States, ${ }^{2}$ Harvard University, Cambridge, Massachusetts, United States, ${ }^{3}$ Harvard University, Somerville, Massachusetts, United States

Periodic lattice distortions (PLD) are at the heart of correlated electronic behaviors such as superconductivity [1], metal-insulator transitions [2], and charge density waves (CDW) [3]. PLDs are typically intrinsic to a crystal [3, 4], Fermi-surface driven [5], accompanied by a CDW, and have periodicity spanning a few unit cells $(\sim 1-2 \mathrm{~nm})$. Recently, extrinsic van der Waals (vdW) driven superlattices with tunable periodicity (up to a few 100nm) were discovered in twisted bilayer graphene (tBLG) [6]. tBLG has been spotlighted for extraordinary correlated electron behaviors at the so-called "magic" angle $\left(1.1^{\circ}\right)$ [7]. Therefore, a full atomistic structural understanding is key to harnessing the exotic properties of tBLG. Here, we provide an analytic description of tBLG superlattices at and near the magic angle using a torsional PLD and report the torsional PLD amplitude of $7.8 \pm 0.6 \mathrm{pm}$ and $6.1 \pm 0.4$ pm for twist angle $(\theta)$ of $1.1^{\circ}$ and $1.2^{\circ}$.

Surprisingly, electron diffraction patterns of tBLG at $1.1^{\circ}$ and $1.2^{\circ}$ show superlattice peaks decorating Bragg peaks pairs [6] — especially more pronounced at higher order Bragg peaks (Fig. 1a). Strong superlattice peaks at high-order Bragg spots are a signature for PLDs. The azimuthal intensity distribution implies transversity of the distortion wave. For unrelaxed tBLG, the diffraction pattern is a trivial superposition of two sets single layer graphene diffraction pattern [8] and shown by quantum mechanical multislice simulation in Figure $1 \mathrm{~b}$. In real space, tBLG forms a moiré pattern with periodicity $\sim \mathrm{ag}_{\mathrm{g}} / \theta$, where ag is the graphene lattice constant $(2.46 \AA)$. The moiré forms regions of energetically favorable AB/BA stacking (Fig. 1d blue, Fig. 1e) and less favorable AA stacking (Fig. 1d red, Fig. 1f). The vdW interaction between layers strives to locally twist (anti-twist) AA (AB/BA) regions to minimize (maximize) interlayer registration and reduce the total interaction energy.

The in-plane structure of magic angle tBLG is described by a torsional PLD field with a single amplitude coefficient $\left(\mathrm{A}_{1}\right)$. The torsional field is made from three non-orthogonal, transverse PLDs with a single harmonic (Fig. 2a):

$$
\left.\Delta=\mathrm{A}_{1}\left(\hat{\mathbf{A}}_{100} \sin \left(\mathbf{q} 100 \cdot \mathbf{r}_{0}\right)+\hat{\mathbf{A}}_{010} \sin \left(\mathbf{q} 010 \cdot \mathbf{r}_{0}\right)+\hat{\mathbf{A}}_{001} \sin \left(\mathbf{q}_{001} \cdot \mathbf{r}_{0}\right)\right) ; \quad \mathbf{A} \perp \mathbf{q} \quad \text { (Eq. } 1\right)
$$

$\mathbf{r} 0, \hat{\mathbf{A}}, \mathbf{q}$ denotes undistorted atom positions, PLD displacement direction, and PLD wavevector. Three q's are $120^{\circ}$ apart. The displacement field in Fig. 2a illustrates the behavior for tBLG relaxation-twisting AA regions in one directions and AB/BA regions in the other. The relaxed structure (Fig. 1c) —obtained by applying displacement to original atomic sites (Fig. 1d) — acts to maximize the low energy regions with $\mathrm{AB} / \mathrm{BA}$ stackings and decreases the high energy regions with AA stacking.

We report torsional PLD amplitude $\left(\mathrm{A}_{1}\right)$ of $7.8 \pm 0.6 \mathrm{pm}$ for $\theta=1.1^{\circ}$ and $6.1 \pm 0.4 \mathrm{pm}$ for $1.2^{\circ}$ magic angle tBLG. The PLD amplitudes were quantified by matching experimental and simulated diffraction intensities. Both experimental and simulated Bragg and satellite diffraction peaks were fitted with sum of 6 parameter 2D Gaussian peaks and constant background term. Relative intensities of selected superlattice peaks to Bragg peaks are plotted against PLD amplitude. Experimental relative superlattice peak intensity 
of $1^{\text {st }}, 2^{\text {nd }}$ and $3^{\text {rd }}$ order Bragg peaks shows great match for determined PLD amplitude (Fig. 2h). Electron diffraction patterns were simulated by Kirkland's multislice package [9]. The error bar represents 95\% confidence interval for least square fit of experimental data to simulation.

The structure of tBLG is a complex moiré material where relaxation between layers acts to minimize high energy AA regions and maximize low energy $\mathrm{AB}$. The atomic rearrangement is remarkably well described with a single coefficient in a single harmonic torsional PLD that can be extracted from a single experimental diffraction pattern.
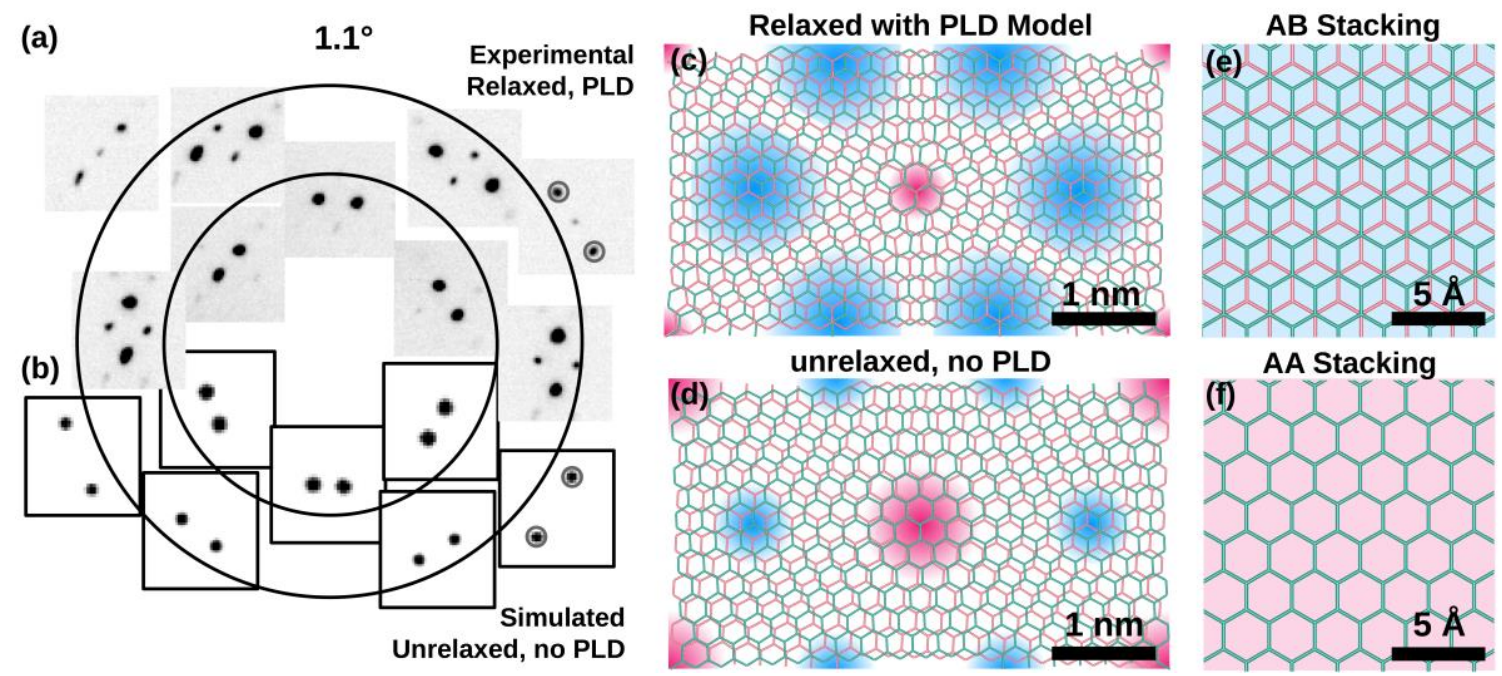

Figure 1. Relaxation of twisted bilayer graphene (tBLG) $\mid$ (a) Electron diffraction of tBLG $\left(\theta=1.1^{\circ}\right)$ displays superlattice peak complexes in addition to two sets of Bragg peaks, marked by gray circles. (b) The simulated diffraction pattern of unrelaxed tBLG only shows two sets of Bragg peaks. The moiré supercell crystal structures of (d) unrelaxed tBLG and (c) relaxed tBLG with PLD model. Red and blue overlays highlight energetically unfavorable AA stacked region, and stable AB/BA stacked region, respectively. PLD decreases the total energy of the system by expanding AB/BA domain and decreasing AA domain. Crystal structure of (e) AB stacked and (f) AA stacked bilayer graphene are shown as reference. (a) is adapted from [1].
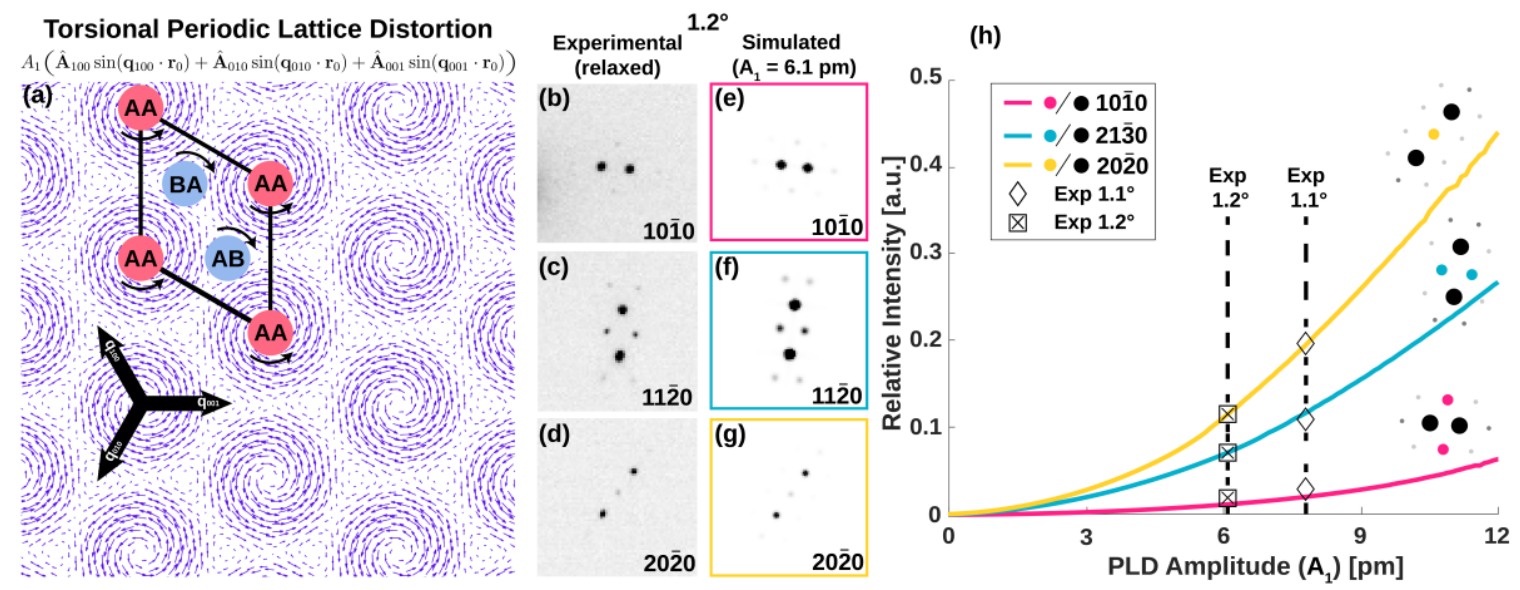

Figure 2. Torsional periodic lattice distortion (PLD) Model | (a) Displacement field of torsional PLD. Local torque directions for AA region and AB/BA regions are opposite. Multislice simulation (e), (f), (g) for PLD with only single harmonic (A1 = 6.1 pm) matches greatly with the experimental data (b), (c), (d). 
(h) Relative intensities of superlattice peaks to Bragg peaks of 1st order (red), 2nd order (blue), and 3rd order (yellow) diffraction spots characterized for a range of PLD amplitude. The plot reveals that $1.1^{\circ}$ and $1.2^{\circ} \mathrm{tBLG}$ are relaxed by single harmonic torsional PLD with amplitude $7.8 \mathrm{pm}$ and $6.1 \mathrm{pm}$, respectively.

References

[1] Sipos, B. et al., Nature Materials, 7, 960-965 (2008)

[2] Mott, N. F. et al., Rev. Mod. Phys., 40, 677 (1968)

[3] Wilson, J. A. et al., Adv. Phys., 24, 2, 117-201 (1975)

[4] Savitzky, B. H. et al., Nature Communications, 81883 (2017)

[5] Peierls, R., Ann. Phys., 396: 121-148 (1930)

[6] Yoo, H. et al., Nature Materials, 18, 448-453 (2019)

[7] Cao, Y. et al., Nature, 556, 43-50 (2018)

[8] Sung, S. H. et al., Phys. Rev. Materials, 3, 064003 (2019)

[9] Kirkland, E. J., "Advanced Computing in Electron Microscopy" $2^{\text {nd }}$ edition (2010) 